\title{
Le 18 Brumaire en Grande-Bretagne : le témoignage de la presse et des caricatures
}

\section{Pascal Dupuy}

\section{(2) OpenEdition \\ 1 Journals}

Édition électronique

URL : https://journals.openedition.org/ahrf/303

DOI : 10.4000/ahrf.303

ISSN : 1952-403X

Éditeur :

Armand Colin, Société des études robespierristes

\section{Édition imprimée}

Date de publication : 1 décembre 1999

Pagination : 773-787

ISSN : 0003-4436

\section{Référence électronique}

Pascal Dupuy, "Le 18 Brumaire en Grande-Bretagne : le témoignage de la presse et des caricatures », Annales historiques de la Révolution française [En ligne], 318 | octobre-décembre 1999, mis en ligne le 12 avril 2006, consulté le 23 avril 2022. URL : http://journals.openedition.org/ahrf/303 ; DOI : https:// doi.org/10.4000/ahrf.303

\section{Ce document a été généré automatiquement le 23 avril 2022}

Tous droits réservés 


\title{
Le 18 Brumaire en Grande- Bretagne : le témoignage de la presse et des caricatures
}

\author{
Pascal Dupuy
}

1 Fin 1798, la Grande-Bretagne prend conscience qu'elle vient d'échapper aux répercussions les plus violentes de la Révolution française. En premier lieu, le départ de Bonaparte pour l'Égypte semble momentanément écarter le danger d'une descente française. Ensuite, l'Irlande radicale et révolutionnaire ne se remet qu'avec beaucoup de difficulté de l'échec de la rébellion des United Irishmen. Conscient de l'étroitesse de la marge de manœuvre qu'il possède, Pitt en profite alors pour accentuer son contrôle de l'opinion publique et de la presse britannique. La censure règne sans partage, l'autorité est rétablie. La confiance change de camp et les radicaux britanniques s'enferment dans un pessimisme empreint de désespoir. Tierney, le nouveau leader des whigs, s'oppose à la politique de Pitt, mais son parti ne représente plus, à cette date, une force d'action susceptible d'incarner l'alternance. Quant à l'opposition foxiste, en s'auto-excluant de la vie parlementaire, elle s'est éloignée des grandes décisions politiques. Sur le plan international, diplomatique et militaire, la victoire navale de Nelson à Aboukir, le 1er août 1798, en reprenant le contrôle de la Méditerranée, a permis à l'Angleterre de se rapprocher de la Turquie et de la Russie du tsar Paul Ier. Cette seconde coalition embryonnaire et dirigée contre la France allait bientôt se renforcer avec l'arrivée de l'Autriche. Le printemps 1799 paraît donc annoncer des jours meilleurs. Toutefois, la situation évolue rapidement dans le sens contraire des intérêts britanniques. Tout à leurs différences, les membres de la coalition se divisent, oubliant les leçons de 1793. La Prusse, malgré les négociations et la venue à Berlin de Thomas Grenville, le propre frère du ministre des Affaires étrangères, refuse toujours obstinément de s'engager du côté des alliés dans la guerre contre la France. L'économie du pays, bien que dopée dans quelques secteurs par le conflit, donne des signes de fatigue. La période 1799-1801 témoignera de graves difficultés d'approvisionnements alimentaires et de famines qui rappellent celles des années 1795-1796. Enfin, le débarquement anglo-russe en Hollande tourne à la déroute et à la débandade et fragilise davantage la politique extérieure du 
Premier ministre anglais. C'est dans ce contexte difficile qu'intervient en France le retour de Bonaparte et le coup d'État du 18 Brumaire dont nous présentons ici les réactions publiques et polémiques en Angleterre appréhendées au travers de la presse et des caricatures.

Une nouvelle révolution?

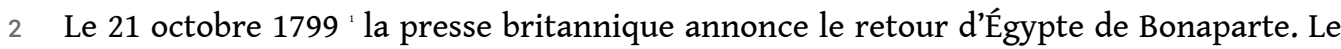
lendemain, le True Briton revient sur les motifs de l'arrivée en France du Général. Il précise qu'à l'inverse des raisons invoquées par le Directoire, Bonaparte se trouvait dans une situation doublement difficile d'un point de vue militaire et diplomatique, son avantage lors de la bataille terrestre d'Aboukir, le 25 juillet 1799, lui permettant simplement, d'après le quotidien, de transformer « une fuite honteuse en un départ victorieux ». Quelques semaines plus tard, le London Packet raconte l'effet sur Sieyès du retour en France de Bonaparte. Le journal révèle ainsi que le futur Consul provisoire, rayonnant, avait demandé à Baudin des Ardennes de deviner les raisons de son humeur joyeuse. Baudin avait suggéré que Sieyès venait d'apprendre une victoire de Championnet? "Mieux que cela» avait-il répliqué. "Une nouvelle victoire de Masséna? L'arrestation de Suvarov? Bien mieux. Les Anglais chassés de Hollande? Encore mieux. Pourrait-il s'agir, demanda Baudin, de la signature des préliminaires de paix ? Non, tu n'y es pas tout à fait, répondit enfin Sieyès, mais c'est un événement qui permettra, peut-être, de l'atteindre ; il s'agit du retour en France de Bonaparte " ${ }^{2}$. Cet événement, et le coup d'État qui s'en suivit se déroulent en Angleterre dans un contexte d'intérêt soutenu pour les affaires françaises et européennes. Ainsi, le 9 novembre, le Sun annonce la publication d'un ouvrage portant sur la Campagne italienne de Bonaparte en 1796-1797 et la traduction imminente d'un livre sur celle de Jourdan de 1799. Deux jours plus tard, le même quotidien insère plusieurs annonces faisant part de la publication en anglais des Annales de la Révolution française de Bertrand de Moleville, d'un récit du siège de Saint-Jean-d'Acre par Bonaparte, d'un compte rendu détaillé de l'expédition en Syrie, de lettres de Sir Sidney Smith rédigées au bord du Tigre et enfin d'un recueil sur la vie du général Hoche. Tout au long de l'automne 1799, on relève dans la presse britannique de longs passages sur l'armée d'Égypte ainsi que des détails sur l'expédition de Syrie et sur la bataille d'Aboukir. La presse juge, comme le gouvernement anglais, que ce qui est en train de se dérouler au Proche-Orient touche à la survie de son commerce avec les Indes et s'intéresse donc intensément, en cette période de crise économique, aux enjeux, aux implications et aux formes mêmes de l'expédition. D'autant que cet intérêt reprend, en écho, la propagande psychologique orchestrée par Sidney Smith depuis le mois d'avril 1799 '. En outre, cette profusion d'informations permet également de masquer partiellement les problèmes rencontrés par les armées anglo-russes en Hollande. L'expédition, après avoir suscité l'espoir, a entraîné dans l'opinion publique une profonde amertume. Le 18 octobre, le Sun s'en prend ainsi au Morning Chronicle, coupable d'amitiés radicales et foxistes, mais aussi d'avoir insinué que la population hollandaise avait accueilli les armées françaises par des manifestations de joie. L'organe du gouvernement reproche au Morning Chronicle de faire semblant de ne pas comprendre les implications stratégiques de l'échec de l'expédition anglaise et de faire ainsi le jeu des jacobins français et anglais. Le Times, pourtant tout à fait docile aux ordres du gouvernement, essuiera, un peu plus tard les mêmes flèches: «avoir aggravé de manière répétée et jacobine les circonstances et l'ampleur des difficultés anglaises en Hollande » '! Le 11 novembre, le Sun abandonne 
ses attaques contre les soi-disant jacobins anglais et se concentre sur l'état intérieur de la République française qui, "malgré ses victoires extérieures, doit faire face à de redoutables scènes d'insurrections " '. Suit une longue liste détaillée de révoltes rurales et urbaines, de Rennes à Marseille en passant par Angers et la Normandie. Le 14, le même journal s'en prend à une proposition du gouvernement français d'échanger le Duc de Choiseul contre des militaires français prisonniers des Anglais. Bien qu'il n'ait aucune sympathie pour la noblesse française émigrée, le Sun précise que le traitement de cette population par le pouvoir français est tout à fait représentatif de la doctrine républicaine française habitée par la haine, la cruauté et la barbarie.

La nouvelle du coup d'État est signalée dans la presse le 15 novembre, par l'intermédiaire, comme le note Joseph Farington, « des journaux français arrivés hier » '. L'observateur de la vie culturelle anglaise remarque qu'il s'agit visiblement « d'une nouvelle révolution" '. Les journaux, pris de court, enregistrent l'information sans véritablement la commenter. Le Sun, reprenant les informations de la presse française, déclare que «Bonaparte est général de Paris, Moreau commande la Garde du Directoire, le Conseil des Cinq-Cents est à Saint-Cloud, Barras a donné sa démission et tout est tranquille à Paris " '. Le 17, les choses sont toutefois un peu plus claires mais laissent encore des zones d'ombres et d'interrogations. Le True Briton parle alors des «convulsions » ayant eu lieu à Paris et commente les nominations de Bonaparte et de Moreau comme des signes de nervosité d'un Directoire se sentant en danger ou voulant faire acte d'autorité. Toutefois, rapidement, l'intérêt se porte sur la personne de Bonaparte et sur la possibilité d'une paix négociée. Le True Briton précise ainsi que Bonaparte est complètement sourd à tout sentiment de pitié et que, s'il le faut, le "monstre corse fauchera sans retenue des milliers d'hommes" 'et le quotidien d'expliquer que cette situation pourra dans l'avenir se révéler périlleuse pour le Directoire, qu'il continue à présenter comme le gouvernement du pays. Les journaux, en revanche, ne comprennent pas bien la démission de Barras et publient sa déposition en intégralité. Pour le Times «Bonaparte, son ami, son élève, sa créature, à qui, dit-il avec vérité, il a ouvert "la carrière de la renommée", l'associé de son cœur, et même davantage, Bonaparte l'a trahi et supplanté, vengeant les torts faits à Barthélémy, de Lepeaux, de tous les amis et collègues qu'il [Barras] a trompés et abandonnés " ${ }^{10}$. Le 18, l'ensemble de la presse britannique donne des détails précis sur les événements parisiens et le True Briton conclut par une série de questions sur les conséquences de cette "nouvelle révolution, semblable aux autres dans son objet, prendre le pouvoir, mais différente dans ses formes puisque c'est la première fois que la Représentation Nationale est déchue " ". Ce journal voit dans les nouveaux événements français la possibilité d'un retour monarchique puisque, à l'inverse des autres journées révolutionnaires, "on ne prêta aucun serment anti-monarchiste». Le quotidien s'interroge aussi sur la possibilité d'une paix à laquelle le nouveau pouvoir français semble attaché. Toutefois, Bonaparte, le " nouveau Cromwell », et le Triumvirat serontils en mesure de garantir l'exécution et le respect des traités? Ils feront sans nul doute des propositions mais qui devront être étudiées avec la plus grande attention, « comme toutes propositions émanant des héritiers de toutes les usurpations, de tous les crimes et de toutes les perfidies de la Révolution française " ". Deux jours plus tard, le True Briton, s'attaque cette fois à l'opposition anglaise intérieure, mais à partir des mêmes arguments. Faussement innocent, le quotidien se demande quelle est la réaction des « avocats de la paix », c'est-à- dire l'opposition et sa presse, à la « nouvelle Révolution 
française " " Que serait-il arrivé si nous avions signé une paix avec les représentants français qui viennent d'être chassés du pouvoir? «Le Cromwellien Bonaparte, et ses sbires n'ont que le mot Paix à la bouche, mais il est fort probable que si cette paix avait été signée auparavant, elle aurait été reprochée à ceux qui viennent d'être éjectés de leur siège. »"

Rapidement, les affaires françaises sont perçues et analysées à travers le prisme de l'histoire anglaise et des intérêts britanniques. On se demande toutefois si le coup d'État était préparé de longue date ou s'il fut réalisé dans la plus grande improvisation? Pour le Sun, il ne fait aucun doute que le coup de force ait été préparé "depuis plusieurs mois » ${ }^{\text {is }}$ par Sieyès qui n'avait pas une aura militaire suffisante pour exécuter son projet seul et qui pour cette raison a fait rappeler Bonaparte en France. Le journal évoque les différents gouvernements qui, depuis 1789, ont présidé aux destinées de la France. Il insiste sur la disparition de la représentation nationale et sur la diminution du nombre des hauts gouvernants, "un Comité de dix [sic] après 1792, puis cinq Directeurs et à présent un triumvirat " ${ }^{16}$. Le 19 novembre, plusieurs journaux publient, traduits en anglais, les dialogues entre un membre du Conseil des Anciens et un membre du Conseil des Cinq-Cents parus dans le Journal de Paris, le 19 brumaire ". En fait, l'Angleterre semble s'interroger sur la qualification à donner au coup d'État: politique ou militaire? Le London Packet, après avoir donné, comme l'ensemble de la presse britannique, un compte rendu détaillé du déroulement des journées, propose ses conclusions. Pour lui, «la dernière Révolution française est entièrement militaire; 10000 soldats et 6 généraux ont suffi à renverser la Constitution ». Bonaparte semble être l'arbitre suprême, un dictateur en devenir, « le seul Maître de Paris, et il est en son pouvoir d'usurper définitivement l'autorité suprême ou de la remettre à qui il veut »". Coup d'État militaire indique le journal, mais pour quoi faire ? Comme dans les milieux royalistes en France ", la possibilité d'un retour des Bourbons est à l'ordre du jour. Canning et d'autres en Angleterre, "considéraient l'avènement de Bonaparte comme une phase d'attente et envisageaient même la restauration imminente de la monarchie dont "l'usurpateur", nouveau Monk, préparait le retour " ${ }^{20}$. Lord Grenville dans une lettre à Wickham, son agent secret, n'envisage guère d'autres solutions pour Bonaparte que de " se jeter dans les bras des royalistes. " ${ }^{2}$

5 Le 20 novembre, les choses s'éclaircissent et grâce aux informations des journaux français, la presse britannique est en mesure de donner des précisions sur le fonctionnement du Consulat, le nouveau gouvernement de la France. On s'intéresse ainsi à ses premières mesures qualifiées de "populaires ", l'abrogation de la loi des otages et le remplacement de l'emprunt forcé par une "subvention extraordinaire». L'ensemble est apprécié puisque, d'après le Sun, ces abrogations vont dans le sens d'un renforcement de la souveraineté de la propriété. Toutefois on s'interroge toujours sur la direction du régime qui possède à sa tête des hommes qui avaient, deux jours avant de la renverser, prêté serment à la République. En fait, continue le journal, ces changements de directions ne touchent pas seulement le gouvernement mais l'ensemble du système administratif français qui s'est empressé de déclarer à ses concitoyens "que cette nouvelle Révolution allait les rendre heureux et libres " ${ }^{\text {}}$. Le London Packet, prenant acte de la nouvelle composition et des nouvelles formes du régime français, décrit minutieusement le château de Saint-Cloud « qui est à la même distance de Paris que Kew de Londres. [...] Le palais est petit, mais très élégant, délicieusement situé sur une petite colline $"{ }^{3}$. Outre, cet intérêt de détails, la presse 
s'interroge également sur la politique extérieure du triumvirat. L'évacuation des troupes françaises de Hollande est évoquée mais semble susciter des avis contradictoires". En fait, la personnalité de Bonaparte est aussi énigmatique. On reconnaît que Sieyès a eu un rôle prépondérant dans toute l'affaire, mais qu'il est à présent quelque peu dépassé et que le pouvoir est détenu par «une oligarchie militaire » dirigée par Bonaparte, dont l'ambition démesurée risque de l'entraîner sur des chemins « imprudents ». Autre incertitude qui inquiète, et soulignée d'ailleurs par le caractère tout à fait nouveau de la situation, - manque de données et modèle analogique défaillant, s'exclame le True Briton désemparé ! - la réaction des autres généraux qui peuvent être, devant la réussite personnelle de Bonaparte, pris d'une sordide jalousie ${ }^{25}$. En revanche, ce qui semble limpide, c'est "que le Rubicon a été franchi et que la République de Boissy d'Anglas a complètement été enterrée comme celle de Robespierre et de Brissot avant elle " ${ }^{26}$. Sa disparition peut entraîner, en d'autres termes, le retour de la monarchie même si le Bonaparte du 18 Brumaire a tout du Cromwell ${ }^{n}$. Le Times ne croit pas un mot, quant à lui, à la théorie d'un retour de la monarchie de France : «Est-ce que Bonaparte a comploté la rentrée des Bourbons dans les déserts de la Syrie, et conspiré contre la République à Alexandrie et au Caire? Il n'est pas facile d'accréditer un rêve si doux ${ }^{2}{ }^{2}$. La presse, qui publie souvent les mêmes articles sur les questions étrangères, s'intéresse de plus en plus à la personne du Premier Consul. L'intérêt est en particulier ravivé par un article du Moniteur du 23 brumaire qui rend compte de la soi-disant tentative d'assassinat de Bonaparte au Conseil des Cinq-Cents. La presse britannique en reprend le contenu et met en doute la véracité de l'événement ". Le True Briton se demande si Bonaparte veut faire concurrence à Jean Debry ${ }^{30}$, " tous les deux descendent, sans nul doute d'Achille, mais nous espérons que leur point faible n'est pas le talon mais le cou et si nous pouvons nous permettre un jeu de mots, espérons que tous les deux seront châtiés par Paris " " ! Le London Packet s'en prend lui aussi au coup d'État machiavélique dont il continue à attribuer la paternité à Sieyès, mais remarque également que les «trente sept » [sic] jacobins bannis étaient de féroces révolutionnaires coupables "d'avoir commis quotidiennement depuis dix ans des crimes odieux" ". Le journal précise que la seule issue pour le nouveau régime est d'arriver à obtenir le soutien des victimes et des adversaires des jacobins ainsi que l'approbation des révolutionnaires modérés attachés à la Constitution de 1791, ceux qu'il surnomme, les « indifférents ». Il explique ensuite que Bonaparte s'est fait Consul car il ne pouvait pas être nommé Directeur en raison de son âge. Il a donc "rétréci » la Constitution à sa taille et la seule différence avec les révolutions précédentes, c'est "que la guillotine a été laissée derrière le rideau " ". Enfin, ce long article se termine sur un passage tiré de la Guerre des Gaules où César explique qu'en Gaule, «pas seulement dans chaque ville, ni dans chaque faubourg, mais dans chaque foyer, il existe des factions. " "

Bonaparte et la Constitution

6 Vers la fin du mois de novembre, l'intérêt pour la nouvelle Constitution et pour la personne de Bonaparte s'intensifie dans la presse et dans les débats publics. Les caricatures anglaises reflètent cette passion teintée de dérision mais aussi d'introspection. Le personnage de Boney, bientôt universellement célèbre dans la caricature européenne, était déjà en 1799 un acteur régulièrement présent sur la scène satirique anglaise ". Ses faits d'armes, la Campagne d'Italie, bien sûr, mais surtout la Campagne d'Égypte lui ont donné une dimension gigantesque même si sa 
représentation formelle est au contraire empreinte de nanisme ! L'expédition d'Égypte avait ainsi donné lieu en mars 1799 à une superbe gravure de James Gillray, Siège de la Colonne de Pompée, puis à un recueil de six gravures introduit par un frontispice par le même auteur et enfin la reprise de l'une de ces estampes, légèrement altérée, pour le magazine London und Paris. L'ensemble, en langue française et anglaise, raillait abondamment les militaires et surtout les scientifiques français qui participaient à cette expédition dont le caractère exotique ne pouvait que stimuler l'imagination déjà fort débridée de Gillray, le propagandiste à crayons le plus célèbre de la production caricaturale anglaise entre 1780 et 1805 . Après le coup d'État du 18 Brumaire, Gillray revient sur le départ d'Égypte du général par une charge acerbe où « ironiquement escorté par les trompettes de la gloire, Bonaparte quitte l'Égypte à la dérobée » ". La gravure "qu'il faut lire à la lumière des événements français de l'automne 1799 montre un Bonaparte couard en train de s'enfuir honteusement en emportant avec lui des sacs d'or.

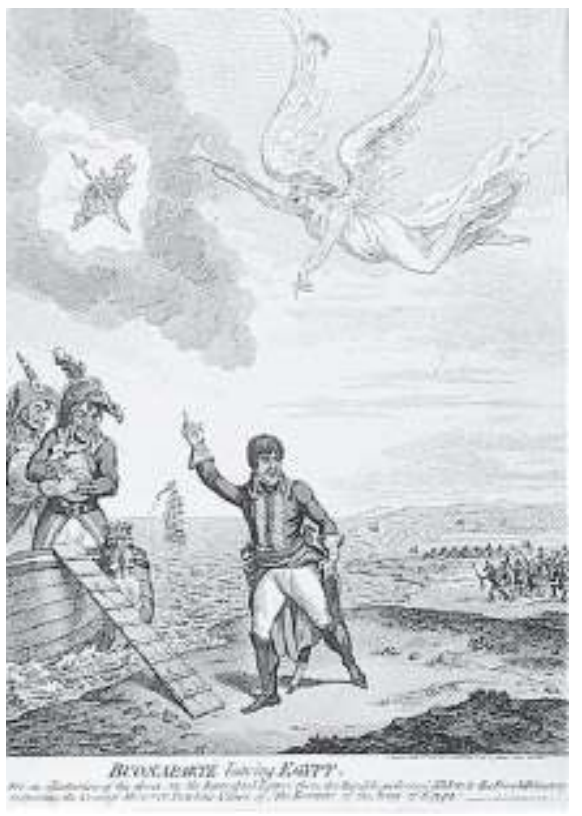

Illustration 1. James Gillray, Buonaparte leaving Egypt, Publiée le 8 mars 1800, eau-forte coloriée, 36,6 x 26,4 cm, Yale Center for British Art, Paul Mellon Collection.

7 D'un air décidé et malin, il s'adresse d'une main pointée en direction d'un faisceau républicain, d'un spectre et d'une couronne, à ses troupes défaites par les Turcs et semble leur dire que les honneurs, la gloire et le pouvoir qui l'attendent en France valent bien mieux que leurs tourments. La tête de Janus à la proue de sa barque souligne son ambition et sa fourberie: son sort personnel passe avant celui de ses soldats. Comme ne l'indique pas cette gravure publiée en mars 1800, les caricatures anglaises réagiront avec célérité aux événements français de novembre 1799. Ainsi, dès le 21, le même Gillray publie une estampe où Bonaparte " met fin à la farce de l'égalité » ". Le général, entouré de ses soldats, lève déjà le bras mais cette fois en direction des députés qui s'enfuient devant cette intrusion et probablement aussi effrayés à l'idée d'être effleurés par cette main menaçante. Pour mieux souligner son propos, Gillray a placé sous la botte de Bonaparte une « liste des membres du Conseil des Cinq-Cents » et en contrepoint, porté par un jeune et simiesque soldat, un tambour où l'on peut lire «Vive la Liberté » que Bonaparte est justement en train d'exécuter. On relève encore 
une caricature "anonyme, non précisément datée mais publiée en novembre, qui présente Bonaparte et ses soldats, tous zoomorphisés en crocodiles, en train de pénétrer dans la salle du Conseil des Cinq-Cents. Comme dans la gravure précédente, tous les députés - représentés sous la forme de grenouilles - s'enfuient, sauf un minuscule batracien qui vient en brandissant son poignard menacer dérisoirement le futur Premier Consul. La scène renvoie, bien sûr, à la soi-disant tentative d'Aréna, le député corse, de poignarder Bonaparte dont on a vu que les journaux anglais s'étaient déjà gaussés. Si les grenouilles commencent à cette époque, après avoir été surtout associées aux Hollandais, à symboliser graphiquement les Français, le crocodile, lui, déjà utilisé par les caricaturistes anglais pour suggérer les pièges égyptiens qui attendaient les scientifiques français, représente un animal préhistorique qui remonte «à l'enfance inquiète de l'humanité; sa vie dans les boues glauques en font un animal fabuleux, incarnation facile du mal et des pulsions les plus obscures, opposant sa laideur sinistre à ceux qu'il menace. " "0

8 Tout aussi rapidement que dans la presse, l'événement est perçu par les caricatures avec une étonnante lucidité et anticipation comme la prise du pouvoir d'un seul homme, en marche vers un régime despotique. On note ainsi le 4 décembre une autre gravure "anonyme où un groupe de pauvres hères agenouillés rend hommage à Bonaparte et lui remet une couronne, symbole de sa prise de pouvoir et des nouvelles fonctions qu'on lui devine.

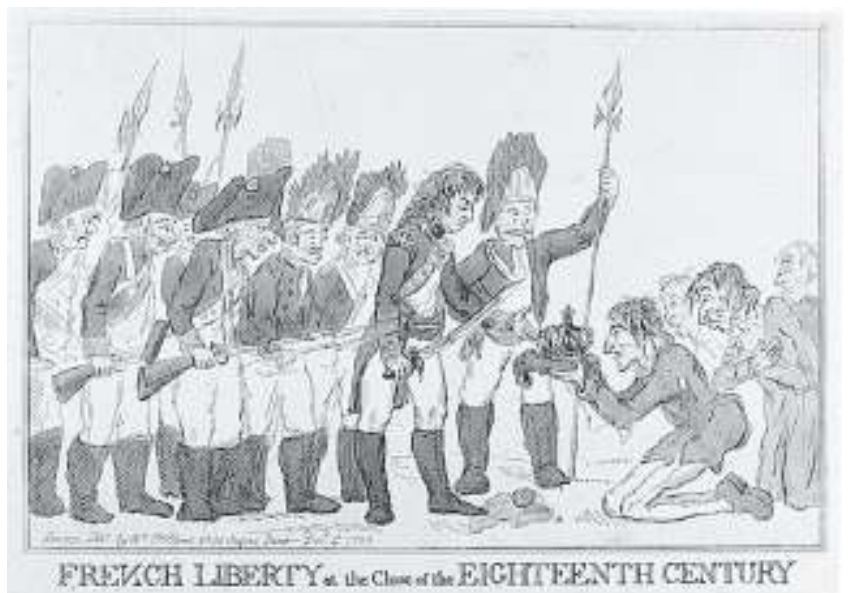

Ilustration 2. Anonyme, French Liberty at the close of the Eighteenth Century, Publiée le 4 décembre 1799, eau-forte coloriée, 28,4 × 39,8 cm, Yale Center for British Art, Paul Mellon Collection.

Il est à noter que ces deux dernières caricatures sont publiées par un éditeur, William Holland, proche des milieux radicaux anglais et qui avait, pour cette raison, un commentaire satirique décalé par rapport à l'ensemble de la production anglaise du moment ". On sent dans ces deux œuvres, à la différence des autres caricatures anglaises, une certaine tristesse, un regard amer jeté sur une illusion et une appréhension de l'avenir. John Cawse (1779?-1862), peintre, occasionnellement caricaturiste, auteur, lui aussi, d'une gravure sur la prise de pouvoir de Bonaparte, n'est en rien proche des milieux radicaux mais au contraire un loyaliste fervent. Il porte ainsi le 30 novembre un regard très amusé sur le coup d'État du 18 Brumaire. 


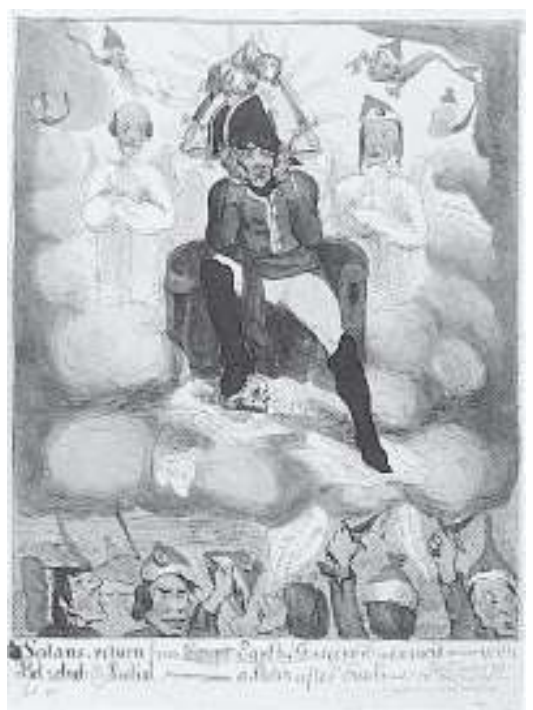

Illustration 3. John Cawse, Satans, returns from Egypt earth. Discovered in Council, with Belzebub \& Belial. A sketch after Fuseli !!!, Publiée le 30 novembre 1799, eau-forte coloriée, 35 × 24,5 cm, Yale Center for British Art, Paul Mellon Collection.

Afin d'introduire son discours, il renvoie dans le titre de sa gravure "à l'œuvre de Johann Heinrich Füssli, dont on sait qu'il fascina James Gillray ", probablement en raison du fait qu'il était, comme l'indiquait son ami, Lavater, qui, lui aussi, exerça une influence majeure sur les caricaturistes, le peintre « de la sauvagerie guerrière et de la sublimité " ". Cawse a choisi ici de représenter à travers le coup d'État le caractère obscur et maléfique de l'inspiration du peintre. Bonaparte, assis sur le trône des enfers, entouré de Sieyès et de Ducos est accueilli par des soldats qui chantent les louanges de Babeuf et qui applaudissent leur nouveau maître. Autour de Premier Consul volent des petites créatures sataniques dont les têtes représentent les leaders de l'opposition foxiste et radicale (Fox, Sheridan, M. A. Taylor et Stanhope). L'ensemble reprend la propagande habituelle de la caricature contre-révolutionnaire anglaise qui mélange avec dérision, mais avec soin, les symboles les plus cinglants de la Révolution française, choisis parmi les plus révulsifs, et les partisans anglais de la paix avec la France ou des réformes constitutionnelles. C'est d'ailleurs ce dernier point qui fait l'objet d'un débat satirique soutenu dans les gravures, comme dans la presse. La multiplication des constitutions en France depuis 1791 est appréciée d'Angleterre comme une hérésie française, une instabilité politique archaïque qui contraste avec la force et la longévité de la Constitution anglaise. Dans cet esprit, nous pouvons nous attarder sur une gravure de Gillray qui, grâce au talent de l'artiste, met en relief de manière éloquente cette attitude. The French Consular Triumverate "représente Bonaparte, Sieyès et les deux consuls (Cambacérès et Le Brun) en train de rédiger la "Nouvelle Constitution » française. 


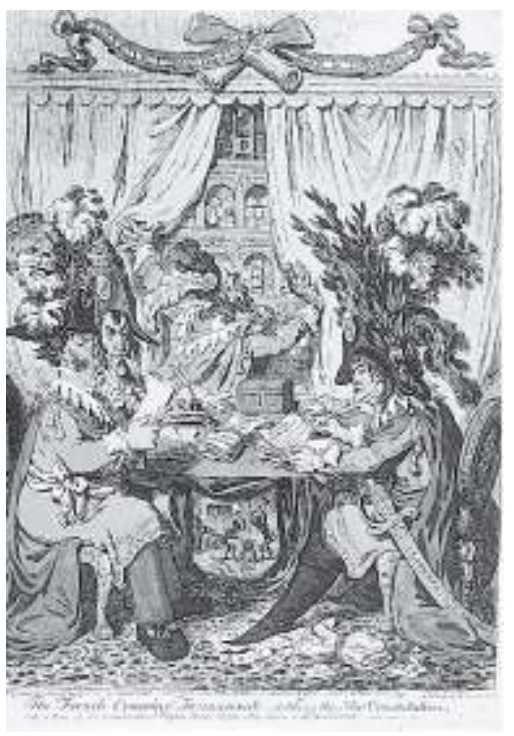

Ilustration 4. James Gillray, The French Consular-Triumverate, settl'ing the New Constitution, with a Peep at the Constitutional-Pigeon-Holes of the Abbe Seiyes in the Back Ground, Publiée le 1er janvier 1800, eau-forte coloriée, 35,7 × 24,9 cm, Yale Center for British Art, Paul Mellon Collection.

11 Gillray, afin de renforcer sa rhétorique, illustre un passage entier d'un pamphlet d'Edmund Burke de janvier 1796 où l'Irlandais répondait aux attaques dont il avait été l'objet à propos de la pension annuelle qui venait de lui être accordée par le roi. Il y déclarait que «L'abbé Sieyès a un vaste colombier dont toutes les cases sont pleines de Constitutions sortant de la main de l'ouvrier, étiquetées, numérotées et assorties pour toutes les saisons et toutes les fantaisies " ". La caricature représente concrètement les consuls et Bonaparte en train de rédiger la nouvelle Constitution et surtout Sieyès en train d'en chercher une dans un colombier caché derrière un rideau. Son choix est rendu difficile en raison des nombreuses constitutions « de Parade, du Sang, de Foutre, de Despotisme... " "qui y sont méticuleusement rangées. L'idée de la prédominance de Bonaparte au sein de la République consulaire est admirablement signalée dans la gravure par une série de signes extérieurs. Ainsi son habit dépasse en magnificence et en ridicule celui de ses collègues, il semble être le seul rédacteur d'une Constitution qui va lui permettre d'accéder au statut de «Grand Monarque», il foule au pied la Constitution de 1793 et les Droits de l'homme, son énorme sabre, la «Liberté », et des diablotins en train de forger des fers indiquant métaphoriquement la direction prise par le nouveau régime: dans cette image le général prépare matériellement sa dictature. Enfin, la gravure est signée «Gillray Fecit» ce qui signifie qu'il ne l'a pas inventée et qu'elle lui fut probablement suggérée par Georges Canning, sous-secrétaire d'État aux Affaires Étrangères et futur Premier ministre ". Cette œuvre illustre parfaitement les liens qui existent en Angleterre entre le pouvoir et la littérature politique et les œuvres de fiction, graphique et satirique. En particulier, elle reflète l'influence de l'activité pamphlétaire de Burke qui, en raison de sa position sur l'échiquier politique et des positions idéologiques qu'il défendait, eut un énorme retentissement dans les îles britanniques, en particulier grâce au relais puissant de la presse. Enfin, la gravure, sous couvert de cynisme et de dérision, met en relief la répulsion anglaise pour «les révolutions françaises » et leur subséquente constitution et a contrario la foi inaltérable en la Constitution anglaise. La presse revient continuellement sur le sujet et sur les différences entre les deux systèmes politiques français et anglais. Ainsi, le 27 novembre le True Briton dans un article sur les 
événements de France écrit que «Montesquieu remarqua que la Constitution anglaise était issue de la forêt allemande. La Constitution française quant à elle a été imaginée dans les déserts d'Arabie " ${ }^{\circ}$. Cette situation favorise l'instabilité et une semaine plus tard un entrefilet dans le même journal indique qu'une faction nouvelle va probablement renverser prochainement celle qui est actuellement au pouvoir. La seule solution, continue le quotidien, serait le retour de la monarchie « mais les chances pour qu'un tel événement se produise n'ont rien de probable. " "st

12 Les journaux proches du pouvoir, comme la quasi-totalité de la production satirique graphique, agissent comme des porte-voix publics en faveur du gouvernement anglais. Ils rendent compte de ses décisions, les commentent, les appuient et sont souvent amenés à les défendre. Inquiets de la tournure des événements en France qu'ils n'avaient pas prévue, Pitt, Grenville, Dundas et l'ensemble des dirigeants anglais rejettent un coup d'État qui a placé à la tête du pays un militaire qui, s'il a perdu une partie de sa réputation en Égypte, n'en reste pas moins un stratège menaçant pour la sécurité du royaume anglais et surtout pour l'équilibre européen ". Refusant de dialoguer avec un "usurpateur", le gouvernement anglais veut établir la solidité du régime avant d'entamer des négociations de paix. Il reproche au nouveau régime sa prise de pouvoir abrupte, sa concentration des pouvoirs et s'inquiète de l'ambition de celui qui semble diriger autoritairement le pays. La presse avec malice, les caricatures de manière satirique vont mettre en avant ces défauts tout en déchargeant au passage leur haine et leur acrimonie contre ceux qui, en Angleterre, osent s'élever contre le gouvernement anglais où se permettent d'émettre des opinions radicales. La presse d'opposition est, quant à elle, désemparée par le coup d'État et le Morning Post ou le Morning Chronicle protestent ainsi contre ce qu'ils ressentent comme une trahison politique et idéologique. Toutefois rapidement, ils reconnaissent également que d'un relatif mal peut naître le bien sous la forme de la paix. Fox, d'ailleurs, sera profondément choqué en janvier 1800 de l'attitude du gouvernement de Pitt de refuser la proposition de paix de Bonaparte ". De distante, l'opposition foxiste et radicale tombera sous le charme, du moins momentanément, du futur Empereur et, après le traité d'Amiens, se pressera en France pour le rencontrer ". Les réactions publiques au 18 Brumaire en Grande-Bretagne sont donc marquées par les enjeux politiques intérieurs anglais et par les considérations diplomatiques et militaires européennes. Elles sont également empreintes de références anglo-anglaises à l'histoire britannique et plus particulièrement à sa Constitution. Finalement, malgré leurs différences, opposition et gouvernement s'accordent sur un point: le 18 Brumaire a mis fin au désordre et au tumulte populaire et politique dans une France qui s'est ainsi rapprochée du modèle britannique. Toutefois, ce modèle issu de cette "nouvelle révolution », dont nous parlent les journaux, n'est pas exempt de défauts. Les réactions instantanées de la presse et des caricatures préfigurent la phase d'incertitude et d'atermoiement des autorités anglaises face à la prise du pouvoir de Bonaparte. Si le gouvernement anglais, en raison des graves difficultés économiques que connait le pays et du coût financier d'une coalition qu'il entretient, se doit de réfléchir à des négociations avec la France, celles-ci ne peuvent s'envisager que dans un contexte militaire favorable à la Grande-Bretagne. Or, en novembre 1799, ces conditions ne sont pas réunies. En outre, plus qu'un avatar supplémentaire, comme veulent le faire croire la presse et les caricatures, d'une histoire française faite de despotisme, d'arbitraire, de violence et d'insurrections, la prise du pouvoir de Bonaparte rappelle aux Anglais celle 
d'un autre chef de guerre, Cromwell, auteur, lui aussi d'une nouvelle constitution. À la fois exotique, puisque se déroulant dans un pays qui semble politiquement profondément éloigné de l'Angleterre, mais également assimilable à un pan détesté de l'histoire anglaise, le 18 Brumaire apparaît doublement condamnable tout en étant cependant un moyen, après réflexions, de parvenir à une paix négociée. Si le traité d'Amiens n'aboutit finalement qu'à une trêve, c'est que le 18 Brumaire contenait, vu d'Angleterre, encore trop d'objets de héritage révolutionnaire détestable et détesté.

\section{NOTES}

1.Voir The True Briton, 21 octobre 1799.

2.The London Packet, or, New Lloyd's Evening Post, 11-13 novembre 1799. Traduction de l'auteur.

3.Henry LAURENS, L'expédition d'Égypte, 1798-1801, Paris, Le Seuil, 1997, pp. 276-277.

4.The Sun, 11 novembre 1799. Traduction de l'auteur.

5.Id.

6.Joseph FARINGTON, The Farington Diary, Londres, Hutchinson \& Co., 1920, vol. I., p. 273. Traduction de l'auteur.

7.Id.

8. The Sun, 15 novembre 1799. En français dans le texte.

9.The True Briton, 17 novembre 1799. Traduction de l'auteur.

10.The Times, 18 novembre 1799. Cité in Jules DECHAMPS, Les îles britanniques et la Révolution française (1789-1803), Bruxelles, La Renaissance du Livre, 1949, p. 121.

11.The True Briton, 18 novembre 1799. Traduction de l'auteur.

12.Id.

13.The True Briton, 20 novembre 1799. Traduction de l'auteur.

14.Id.

15.The Sun, 18 novembre 1799. Traduction de l'auteur.

16.Id.

17.The Sun, 19 novembre 1799.

18.The London Packet, or, New Lloyd's Evening Post, 18-20 novembre 1799. Traduction de l'auteur

19.Voir Jean-Paul BERTAUD, Bonaparte prend le pouvoir, Bruxelles, Éditions Complexe, 1987, pp. 169-172 et Alphonse AULARD, "Le lendemain du Dix-Huit Brumaire", in Études et Leçons sur la Révolution française, Paris, 1911, pp. 225-228

20.Jules DECHAMPS, op. cit., p. 122.

21.Id.

22.The Sun, 20 novembre 1799. Traduction de l'auteur.

23.The London Packet, or, New Lloyd's Evening Post, 20-22 novembre 1799. Traduction de l'auteur.

24.Id.

25.The True Briton, 22 novembre 1799. 
26.Id.

27.Id.

28.The Times, 25 novembre 1799. Traduction de l'auteur.

29.Sur cette légende bonapartiste voir : A. AULARD, "Bonaparte et les poignards des

Cinq-Cents”, in Études et Leçons sur la Révolution française, Paris, 1902, pp. 271 et suivantes.

30.Jean Debry échappa à un attentat le 28 avril 1799.

31.Pâris guidé par Apollon tua Achille. The True Briton, 23 novembre 1799. Traduction de l'auteur.

32.The London Packet, or, New Lloyd's Evening Post, 25-27 novembre 1799. Traduction de l'auteur.

33.Id.

34.Id.

35.Voir Pascal DuPUY, "La campagne d'Italie dans les gravures anglaises sous le Directoire : diffusion et interprétations (1796-1798)", in Jean-Paul BARBE et Roland BERNECKER (éd.), Les intellectuels européens face à la campagne d'Italie, 1796-1798, Mûnster, Nodus Publikationen, 1999, pp. 209-231.

36.Napoléon vu à travers la caricature, Catalogue du Musée Napoléon d'Aremberg, Zürich, 1998, p. 178.

37.James Gillray, Buonaparte leaving Egypt, Publiée le 8 mars 1800, BMC 9523 et Yale Center for British Art, Paul Mellon Collection B1981.25.995.

38.James Gillray, Exit Liberté à la François! or Buonaparte closing the farce of egalité, at St Cloud near Paris, nov. 10th 1799, Publiée le 21 novembre 1799, BMC 9426. Traduction de l'auteur.

39.Anonyme, The Corsican crocodile dissolving the Council of Frogs!!!, Publiée en novembre 1799 , BMC 9427.

40.Michel JOUVE, "La caricature graphique sociale et politique en Angleterre de 1760 à 1804", Thèse pour le Doctorat d'État s.l.d. de J. Dulck, Université de Paris III, 1979, p. 297. 41.Anonyme, French Liberty at the close of the Eighteenth Century, Publiée le 4 décembre 1799, BMC 9433 et Yale Center for British Art, Paul Mellon Collection B1981.25.1944. 42.Sur l'ensemble de la production satirique graphique anglaise voir Pascal DuPuY, L'Angleterre face à la Révolution : la représentation de la France et des Français à travers la caricature (1789-1802), Thèse de Doctorat, s.l.d. de C. Mazauric, Université de Rouen, 1998, 3 Tomes.

43.John Cawse, Satans, returns from Egypt earth. Discovered in Council, with Belzebub \& Belial. A sketch after Fuseli!!!, Publiée le 30 novembre 1799, BMC 9431 et Yale Center for British Art, Paul Mellon Collection B1981.25.1568.

44. Voir Draper HILL, Mr Gillray, the caricaturist, Londres, Phaidon, 1965, $266 \mathrm{pp}$.

45.Werner HOFMANN, Une époque en rupture, 1750-1830, Paris, Gallimard, 1995, p. 187. Sur les rapports entre la caricature, Füssli et Lavater voir Pascal DuPuY, "Interprétation d'un cauchemar : "The Nightmare" de Füssli, in Ridiculosa, n³, 1996, pp. 111-128.

46.James Gillray, The French Consular-Triumverate, settl'ing the New Constitution, with a Peep at the Constitutional-Pigeon-Holes of the Abbe Seiyes in the Back Ground, Publiée le 1er janvier 1800, BMC 9509 et Yale Center for British Art, Paul Mellon Collection B1981.25.828.

47.Edmund BURKE, Lettre de M. Edmund Burke à un noble lord, Londres, 1796, in Réflexions sur la Révolution de France, Paris, Hachette, 1989, p. 501. 
48.Id. En français dans le texte.

49.Mary Dorothy GEORGE, English Political Caricature. A study of opinion and propaganda, 1793-1832, Oxford, Oxford University Press, 1959, Vol. II, pp. 48-49.

50.The True Briton, 22 novembre 1799. Traduction de l'auteur.

51.Id., 28 novembre 1799. Traduction de l'auteur.

52.Voir Harvey MITCHELL, The underground war against revolutionary France. The missions of William Wickham, 1794-1800, Oxford, Oxford University Press, 1965, pp. 238-239. John M. SHERWIG, Guineas and gunpowder. British foreign aid in the wars with France, 1793-1815, Cambridge, Harvard University Press, 1969, pp. 116-142.

53.L. G. MITCHELL, Charles James Fox, Londres, Penguin Books, 1992, pp. 166-167.

54.Henri FAUVILLE, La France de Bonaparte vue par les visiteurs anglais, Aix-en-Provence, Édisud, 1989, pp. 85-102.

\section{RÉSUMÉS}

Le coup d'État du 18 Brumaire est rapidement connu en Angleterre. La presse l'évoque dès le 16 novembre. Les événements sont relatés, détaillés et s'inscrivent dans un contexte d'intérêt renouvelé pour les affaires françaises. L'opinion publique anglaise, les journaux et les caricatures, relèvent le caractère brusque de la mise en place du régime consulaire mais surtout se gaussent d'une énième constitution mise en place par un tyran.

The coup d'État of 18 Brumaire in Great Britain as viewed by the press and caricatures. The British quickly learned about the coup d'État of 18 Brumaire. As quickly as 16 November, the press responded to the renewed interest in French affairs by relating and detailing the events of the coup. While calling attention to the brusque nature of the consular regime, above all, English public opinion, newspapers and caricatures ridiculed the umpteenth constitution put in place by a tyrant.

Il 18 Brumalo in Gran Bretagna : la testimonianza della stampa e della caricatura. La notizia del colpo di stato di Brumaio si diffonde rapidamente in Inghilterra. La stampa ne parla dal 16 novembre. Gli eventi sono riferiti con dovizia di dettagli, in un contesto di rinnovato interesse per gli affari francesi. L'opinione pubblica inglese, i giornqli e le caricature rilevano il carattere repentino dell'ascesa del regime consolare, ma soprattutto si fanno beffe di un'ennesima coscituzione proclamata da un tiranno.

El golpe de estado del 18 de brumario es conocido muy pronto en Gran Bretaña. La prensa habla de ello a partir del 16 de noviembre. Los acontecimientos son relatados con detalles en un contexto de interes renovado por los asuntos franceses. La opinión pública inglesa, los periódicos y las caricaturas revelan el carácter brusco del advenimiento del régimen del consulado, pero se burla también de la enísima constitución proclamada por un tirano.

Der 18. Brumaire in Großbritanien: Das Zeugnis der Presse und der Karikatur. Der Staatsstreich vom 18. Brumaire wurde in England schnell bekannt. Die Presse erwähnt ihn schon am 16. November. Sie berichtet sehr ausführlich über die Ereignisse und dies zeugt von einem erneuerten Interesse für Frankreichs Angelegenheiten. Die englische öffentliche Meinung, die 
Zeitungen und Karikaturen unterstreichen die Plötzlichkeit der Einrichtung des Konsulats, spotten aber vor allem über eine zum xten Mal von einem Tyrannen in Kraft gesetzte Verfassung.

\section{AUTEUR}

PASCAL DUPUY

Université de Rouen 